\title{
A FIELD STUDY OF THE INDUSTRIAL MODELING PROCESS
}

\author{
B.A. Foss ${ }^{*}$ B. Lohmann ${ }^{* *}$ W. Marquardt ${ }^{* *}$ \\ * Department of Engineering Cybernetics, Norwegian University \\ of Science and Technology, N-7034 Trondheim, Norway \\ ** Process Engineering, RWTH Aachen University of Technology, \\ D-52056 Aachen, Germany
}

\begin{abstract}
This paper analyzes the modeling process by means of a field study in the chemical industries. A diversified set of experienced modelers from two different countries have been interviewed using a case study approach. The interviews focussed on the modeling process. Other issues like life-cycle perspectives and the use of modeling in the process industries were treated as well. The information has been summarized and presented in a structured manner imposing the least possible bias from the authors. Based on the interview information the modeling process is discussed in detail. Further, fundamental research issues are identified, and a research agenda is proposed.
\end{abstract}

Keywords: Process models, dynamic modeling, computer-aided modeling, case study approach

\section{INTRODUCTION}

In order to retain or increase their market share chemical process industries, which face increasing pressure from environmental and safety regulations as well as growing demands on product quality and availability, have to continuously improve process operation and existing chemical processes. Further, new processes need to be developed. Time and cost constraints force these industries to reduce their experimental effort during process development and to facilitate, even routinize, the application of model-based process technology such as model-based production planning and scheduling, or model-based process optimization and control.

Despite the commercially available modeling tools, the effort spent for all kinds of modeling activities is the most time consuming step in an industrial project where model-based process engineering techniques are applied. It is conjectured that this comparatively high effort prevents the application of state-of-the-art model-based technology in many industrial projects.

One important means to overcome this modeling bottleneck is the development of more advanced computer-based tools supporting the modeling process, which can be viewed as a sequence of activities like model generation, validation, documentation, or application. There is significant research activity in various groups aiming at the development of novel computer tools (cf. the reviews of of Marquardt (1996) and Pantelides and Britt (1994) for an overview), but as long as a detailed understanding of the process of model development in industrial practice is missing the productivity of modeling engineers and the quality of models will not be improved sufficiently by more advanced modeling tools.

Analyzing development or business processes is already a major research area in other fields like business process reengineering and workflow man- 
agement (Hammer and Champy, 1995), software engineering (Finkelstein et al., 1994), or Total Quality Management (Oakland, 1989), as the improvement of such processes has a major impact on the performance of industrial companies and the quality of the products being produced. But while e.g. software development processes have been analyzed by empirical studies (Curtis et al., 1988), comprehensive and detailed empirical investigations of the modeling process are not available.

Therefore, this contribution describes an empirical investigation that was carried out in different industrial companies in order to gather information on the current status of the process of model development in industry. It was the intention of the authors to deliver a first contribution to an improved understanding of this modeling process, and finally to come to some suggestions for improving current modeling technology. The investigation focuses on first principles based dynamic models. Hence, black-box modeling and steadystate modeling is only treated in a more peripheral manner.

After a discussion of the research methodology that was applied during the preparation and conduction of the investigation the results of the interviews are summarized. It is intended to provide an objective summary with the least possible bias introduced by the authors' interpretation of the interview information. An assessment of the interviews will be made in the last section, where our understanding of the modeling process and a research agenda for advanced modeling tools are formulated.

\section{RESEARCH METHODOLOGY}

An improved understanding of the modeling process must be based on empirical evidence since there is no established and accepted theory of modeling available (Aris, 1991). This motivates our research methodology.

\subsection{Case-study approach}

Our research methodology is based on a case study approach (Yin, 1984; Carroll, 1995), which is a frequently employed technique in social sciences to acquire empirical data in complex situations. Case-studies are chosen if "... a how or why question is being asked about a contemporary set of events, over which the investigator has little or no control" (Yin, 1984). The modeling process can be viewed as such a contemporary set of events. Moreover, it is a creative poorly understood activity which seems to heavily depend on the individual's background and preferred work process.
Due to the poor understanding and the highly individual characteristics, a generic fine granular task structure, which would be mandatory in many other empirical methodologies such as surveys, cannot be defined in advance. Therefore, a group of domain experts should be faced, during interviews, with a carefully designed and precisely defined case comprising one or more typical modeling problems instead of a questionnaire on the modeling process in general.

Due to the limited availability of experienced modeling practitioners for interviews, the complete solution of a realistic modeling case, taking at least a couple of days to a few months, is impossible. Therefore, a modified case study approach has been chosen. Instead of presenting the same prepared case - the realistic modeling problem - to all of the selected domain experts, we have asked everyone to choose a modeling problem from his personal experience. The solution of the modeling problem will be described and discussed on a common basis during an interview. This modification does not only render the knowledge acquisition process feasible. Rather, it also guarantees that the expert modeler communicates his (and not the interviewer's) work process based on sound and intimate knowledge about the case of his preference. It can be expected, that this approach results in better and more realistic statements on the modeling process.

The drawback of this modified case study approach may be seen in a lack of comparability of the interview results. In order to minimize this potential problem, detailed information has been provided to the interviewees to prepare the interview. This information and other details of the interview preparation are summarized in the following section.

\subsection{Interview preparation}

In order to capture realistic information on the modeling process as carried out in industry at present, a group of people with diversified modeling experience in an industrial setting needs to be carefully selected. The selection should be guided by the general requirement that every interviewee should have his/her own and individual view of the subject in order to get a broad and hopefully unbiased set of data. This also includes, that the interviewees should be selected from different industries regarding size and core business in different countries.

Table 1 summarizes the profile of the 16 interviewees included in the study. The average number of years of modeling experience in industry is well above 10 . The interviewees have university 
degrees, most of them equivalent to a Ph.D. degree in diverse areas such as physics, mathematics, chemical or electrical (control) engineering. All of them are working for a process engineering company, a large operating company or a vendor in Norway or Germany.

Table 1. Profile of interviewees. The last column indicates the main areas of activities: $\mathrm{R}$ - university; $\mathrm{P}$ - chemical process industries; $\mathrm{V}$ - vendor company.

\begin{tabular}{cllrl}
\hline No. & & Education & \multicolumn{2}{c}{ Experience } \\
\hline I1 & Dr.ing. & control engg & $5 \mathrm{y}$ & $\mathrm{R}, \mathrm{P}$ \\
I2 & M.Sc. & chemical engg & $16 \mathrm{y}$ & $\mathrm{V}$ \\
I3 & Ph.D. & chemical engg & $22 \mathrm{y}$ & $\mathrm{V}, \mathrm{P}$ \\
I4 & Dr.ing. & mechanics engg & $11 \mathrm{y}$ & $\mathrm{P}$ \\
I5 & Dr.ing. & chemical engg & $13 \mathrm{y}$ & $\mathrm{P}$ \\
I6 & Siv.ing. & control engg & $15 \mathrm{y}$ & $\mathrm{V}, \mathrm{P}$ \\
I7 & Dr.ing. & physics,control & $18 \mathrm{y}$ & $\mathrm{P}$ \\
I8 & Dr.ing. & control engg & $20 \mathrm{y}$ & $\mathrm{V}, \mathrm{P}$ \\
I9 & Dr. rer. nat. & physics & $17 \mathrm{y}$ & $\mathrm{P}$ \\
I10 & Dr. rer. nat. & physics & $13 \mathrm{y}$ & $\mathrm{P}$ \\
I11 & Dr. rer. nat. & mathematics & $9 \mathrm{y}$ & $\mathrm{P}$ \\
I12 & Dr.-Ing. & electrical engg & $14 \mathrm{y}$ & $\mathrm{V}, \mathrm{P}$ \\
I13 & Dr.-Ing. & chemical engg & $9 \mathrm{y}$ & $\mathrm{R}, \mathrm{P}$ \\
I14 & Dr.-Ing. & chemical engg & $10 \mathrm{y}$ & $\mathrm{R}, \mathrm{P}$ \\
I15 & Dr.-Ing. & electrical engg & $6 \mathrm{y}$ & $\mathrm{R}, \mathrm{P}$ \\
I16 & Dipl.-Math. & mathematics & $25 \mathrm{y}$ & $\mathrm{P}$ \\
\hline
\end{tabular}

Interview information had been prepared and submitted to the interviewees prior to the interview. This document includes information on the background and objectives of the empirical investigations, a sequence of coarse tasks which typically occur in every modeling process, a set of important questions on certain aspects of the modeling process, and an outline of the conduction of the interview.

On the other hand, guidelines for conducting the interviews have been agreed upon by both investigators. The interview guide includes the interview information as provided to the interviewees as well as a number of interview practicalities. The investigators carried out pilot interviews with experienced Ph.D. students of their research group to assess and confirm the interview structure.

\subsection{The modeling process - a task structure}

The finalized task structure used in order to focus the interviews is summarized in the following together with a brief description of the task content for better reference:

(1) Initial data collection and problem formulation. The initial data are collected and some more or less precise formulation of the modeling problem is developed.

(2) Tools selection. The software tools to support modeling and allow simulation are selected.

(3) Conceptual modeling. The plant to be modeled is abstracted at first. The essential compartments and the dominant physicochemical phenomena occurring are identified and documented for later reuse.

(4) Model representation. A representation of the process model is generated. Often, equations are used, however, a graphical block diagram (or any other formalism) may alternatively be used depending on the modeling tools selected above.

(5) Implementation. The model representation is implemented using the means provided by the modeling system of the software employed. These may range from general programming languages, to equation-based modeling languages or graphical block-oriented interfaces.

(6) Verification. The model implementation is verified to really capture the intent of the modeler. No simulations for the actual problem to be solved are carried out for this purpose.

(7) Initialization. Reasonable initial values are provided or computed, the numerical solution process is debugged.

(8) Validation. The results of the simulation are validated against some reference, ideally against experimental data.

(9) Documentation. The modeling process, the model, and the simulation results during validation and application of the model are documented.

(10) Model application. The model is used in some model-based process engineering problem solving task.

This task structure of the modeling process is deliberately of a coarse granularity. Iterations are not taken into account at this stage but deferred to Section 4.1.

\subsection{Interview conduction and evaluation}

One-to-one interviews have been conducted on the basis of the interview guide. The information has been communicated orally and by blackboard or flipover. In most cases, notes have been taken by an assistant of the interviewer, but no tape recording has been used. It was agreed that all details on the modeling case studies will be kept confidential. No written material on the discussed cases has been provided by the interviewee.

The task structure has been successfully used to focus the discussion. Nevertheless, every theme has been discussed in an open-ended manner in order to render the interviewee the freedom possible. The investigators avoided to discuss the facts given by the interviewees. Only clarifying questions have been posed and intermediate summaries have been inserted by the interviewers. 
The evaluation of the interviews is one of the least developed and most difficult aspects in doing conducting studies (Yin, 1984). Hence, only few evaluation methodologies are available in the literature. It is suggested to match the findings from the interviews with previous propositions such as the task structure suggested in the interview information. Further, the findings should be compared to the investigators' expectations which stem from extensively dealing with the subject over the past years. An unbiased comparison has been found to be quite difficult or even impossible because such comparison is typically done during the interview. Statements deviating from the interviewer's expectation typically trigger clarifying questions or the next theme of discussion and therefore influence the interviews significantly.

The investigators' evaluation of the interviews has been compared among each other. Their findings have been consistent to a surprisingly large extent which leads us to significant confidence in the results presented subsequently. In order to definitely exclude any kind of misinterpretation, the study results have been approved by all the interviewees.

In the following summary of the interviews we present exclusively the views of the interviewees, whereas our interpretation is summarized in the following discussion section.

\section{RESUME OF INTERVIEWS}

The scope of this section is to present the information gained from the interviews in a summarized and structured manner. First, the scenario projects are presented. Then, the modeling process is discussed in detail. Thereafter, information gathered on life-cycle perspectives and general aspects on modeling in the process industries are considered before a wish list ends the section.

\subsection{Scenario projects}

The scenario projects are summarized in Table 2 . The listing refers to the interviewees in the first column.

In most cases, only one scenario has been discussed in detail, whereas a second scenario has been briefly summarized in addition and contrasted to the first one. The projects are quite diverse. However, the purpose of most of the projects has been the solution of some chemical or control engineering problem rather than delivering a simulator as in I2, I3, I6, or I11. There is a mix of fundamental nonlinear models and simplified block diagram type models (e.g. I12 and I15) as commonly used in control engineering, although fundamental models have been dominating.

In all projects, model development forms a common thread. Only I13 considered a steady-state flowsheet model whereas a dynamic model has been developed in all other projects. In most cases, the complete process model or some parts of it have been developed from scratch. For example, I1, I4 and I8 focus on unit model development whereas I5, I9, I10, or I11 cover plant-wide modeling from scratch. Reuse and modification has occurred in quite a number of cases. Reuse not only refers to selecting and configuring unit models from a library as typically done in flowsheeting (as in I2, I6, and I13) but also covers the modification of already existing nonstandard unit models (as in I11 and in I12). In particular, a steady-state plant-wide model has been the starting point for dynamic model development in I14.

All the deliverables, except in I3, are one-of-akind. The deliverable in I3 is a general purpose niche simulator intended for the oil and gas industries. Nine of the projects have been initiated by a research centre (technology push projects) whereas twelve have been initiated by the problem owners themselves (market pull projects).

Table 2. Profile of scenario projects. Third column: modeling from scratch $(\mathrm{S})$, reuse and modification $(\mathrm{R})$, modeling on the flowsheet level (F). Fourth column: one or many product applications. Fifth column: technology push $(\mathrm{Tp})$ or market pull $(\mathrm{Mp})$ projects.

\begin{tabular}{|c|c|c|c|c|}
\hline No. & $\begin{array}{l}\text { Project } \\
\text { deliverable }\end{array}$ & $\begin{array}{l}\text { Mod. } \\
\text { type }\end{array}$ & $\begin{array}{l}\text { No. } \\
\text { prod. }\end{array}$ & $\begin{array}{l}\mathrm{Tp} / \\
\mathrm{Mp}\end{array}$ \\
\hline $\mathrm{I1}$ & $\begin{array}{l}\text { operator support } \\
\text { system }\end{array}$ & $\mathrm{S}$ & one & $\mathrm{Tp}$ \\
\hline $\mathrm{I} 2$ & training simulator & $\mathrm{R}, \mathrm{F}$ & one & $\mathrm{Mp}$ \\
\hline I3 & niche simulator & S & many & $\mathrm{Mp}$ \\
\hline $\mathrm{I} 4$ & advanced control & S & one & $\mathrm{Tp}$ \\
\hline I5 & simulator for design & $\mathrm{S}, \mathrm{F}$ & one & $\mathrm{Mp}$ \\
\hline I6 & training simulator & $\mathrm{R}, \mathrm{F}$ & one & $\mathrm{Mp}$ \\
\hline I7 & study results & S & one & $\mathrm{Mp}$ \\
\hline \multirow[t]{2}{*}{ I8 } & model control design & S & one & $\mathrm{Tp}$ \\
\hline & surveillance & $\mathrm{R}, \mathrm{F}$ & one & $\mathrm{Tp}$ \\
\hline \multirow[t]{2}{*}{ I9 } & process design & $\mathrm{S}, \mathrm{F}$ & one & $\mathrm{Tp}$ \\
\hline & equipment design & S & one & $\mathrm{Mp}$ \\
\hline $\mathrm{I} 10$ & process development & S & one & $\mathrm{Tp}$ \\
\hline $\mathrm{I} 11$ & $\begin{array}{l}\text { dynamic simulator for } \\
\text { design and control }\end{array}$ & $\mathrm{R}$ & one & $\mathrm{Mp}$ \\
\hline \multirow[t]{2}{*}{$\mathrm{I} 12$} & control design & S & one & $\mathrm{Mp}$ \\
\hline & safety analysis & $\mathrm{R}$ & one & $\mathrm{Mp}$ \\
\hline I13 & process debottlenecking & $\mathrm{R}, \mathrm{F}$ & one & $\mathrm{Mp}$ \\
\hline $\mathrm{I} 14$ & controllability study & S & one & $\mathrm{Mp}$ \\
\hline $\mathrm{I} 15$ & control design & S & one & $\mathrm{Mp}$ \\
\hline $\mathrm{I} 16$ & on-line optimization & S & one & $\mathrm{Tp}$ \\
\hline
\end{tabular}

\subsection{The modeling process}

In this section we present the results on the model development process as presented to us by 
the interviewees. The interviewees agreed on our proposed task structure, in the sense that all the tasks were present more or less explicitly. The modeling process is, however, by no means linear. It is characterized by extensive iterations which will be discussed later.

\subsubsection{Problem statement, functional specification, and initial data collection}

A precise problem statement including a functional specification of the deliverable should be always part of a project. However, there has been no functional specification of the model in any of the scenarios studied since the model is a part of a model-based application such as a system for plant-wide on-line optimization. Hence, we must distinguish between the functional specification of the application and of the model within the application. Hence, if we assume a specification of the functionality of the application (as most often true for market pull projects), it is also implicitly fixing the specifications on the model which must be inferred for model development.

Sometimes however, there is no sufficiently precise problem statement defining the model-based application. Rather, there is some vague description of the chemical or control engineering problem to be solved. This is very pronounced in technology push projects. These projects may be initiated in different ways. First, a research centre may offer some study on an interesting issue to a plant owner. This results in a series of projects performed by a research centre, usually in cooperation with the plant owner, providing improved process understanding and, in some cases, a series of more and more advanced model-based solutions. Second, a strategic research project may be initiated by the senior management in order to assess new model-based technology like on-line optimization, scheduling etc.

For market pull projects there typically exists a more concrete problem statement which can easily be mapped into a requirements specification of the model-based application (but not to the model explicitly). Some examples are (i) check the control system design for a new process as provided by a contractor and assess control performance, (ii) develop a control system to limit emission to some given target value, or (iii) identify the bottleneck in a process and suggest process modifications to increase capacity. The requirements specification is usually most detailed in the cases where the deliverable is a system like a training simulator provided by a vendor company to a client.

In summary, the specification of model fidelity and functional characteristics of model-based application are typically not stated in sufficient detail or not even available at all at the beginning of a project. Many interviewees state that the model specification becomes more distinct as the project develops and better process understanding has been built up. This implies that model specifications may change during a project even though the functionality of the application is unchanged. By this, development of the specification becomes part of the modeling process itself. Often, model specifications are not made explicit at all. At best, they are inferred from the problem statement.

In addition to the derivation of functional specifications information about the process is collected and basic process understanding is gradually built up in the early phases of a modeling project. It is important to concentrate on facts only; any kind of interpretation by some domain expert should be taken with care. There are numerous sources of information; they include (i) people who know the process or a part of it very well (operating personnel, process or control system designer, maintenance personnel, product quality control people, etc.) at the plant and in the technology centre, (ii) patent and scientific literature, (iii) inhouse process documentation, and (iv) operations log.

A complete set of data for physical property and reaction rate calculations is essential and often not available. An experimental program is sometimes defined and started in the early stages of a project in order to have access to missing data as soon as possible during the course of the project.

The collection of information is driven by the needs of problem solution and therefore continuously carried out during the whole project, though the major effort is located at the beginning of the project. The information gathered is always filtered regarding the purpose of the model.

\subsubsection{Modeling environment}

Always, the choice of the type of modeling environment is made in the very early stages of a project. Often it is part of the requirements definition phase. Typically, a modeler has his preferred set of tools for different applications. There are three types of simulators: a flowsheeting system for (standard) steady-state problems, an equation-oriented simulator for nonstandard steady-state or dynamic problems and a block diagram oriented simulator for control related applications. Niche simulators tailored to a particular application area such as polymer processes or downstream processes in the oil and gas industries are rarely used. Large operating companies try to limit the number of tools used to a manageable limit. Computational fluid dynamics (CFD) codes are increasingly employed for detailed analysis of certain units such as chemical reactors. Hence, in 
general there is a close link between the class of problems and the simulator used.

Most of current modeling and simulation tools lack support for analysis of dynamic models. Examples are time series processing and frequency domain or state space analysis tools. As a consequence, some modelers prefer to use a tool with comprehensive support for model analysis such as Matlab and toolboxes to develop models from scratch. The code generation facility of these tools is used to generate $\mathrm{C}$ or Fortran code which can be integrated into the application.

Usually, tools stemming from the control system design or simulation systems community do not offer any particular support for rigorous thermodynamics computations. Such tools may therefore not be considered if physical properties of complex mixtures need to be computed with significant accuracy. However, some of the interviewees state that this problem can often be circumvented if a simplified local thermodynamic model is sufficient which can easily be coded in the simulation tool. This is always true in control applications, where the limits of the operating range are typically known in advance. Alternatively, an external thermodynamics package may be linked to the process simulation system to build a two-module application (as done in I3). This approach may be resource consuming since it is virtually impossible to specify the external package in sufficient detail to circumvent link-up problems during debugging and validation.

Packages for black box modeling based on system identification techniques are frequently used by those interviewees having a control engineering background. Only in some cases rigorous identification techniques (Ljung, 1987) are employed. More frequently, very simple block diagram models composed of linear dynamic and nonlinear gain elements are derived on physical grounds and roughly adapted to the real process using few experimental or design data. Nonlinear black box models such as neural nets have only been occasionally used in explorative technology assessment studies by some of the interviewees. In these cases dedicated commercial modeling tools have been employed.

The interviews show that the degree of sophistication and the technical set-up of a modeling tool heavily influences the modeling process in general and the work process of the modeler in particular. Modeling tools contribute significantly to increased efficiency of the modeling process. As an example, the provision of model libraries for typical process units has been one of the key success factors for the wide acceptance of steadystate flowsheeting. For facilitating plant-wide dynamic simulation, adequate model libraries are still largely missing. However, for some areas, like in gas and oil processing, library models exist for all important units in dedicated simulation systems. The situation is perceived as very different when nonstandard unit models need to be developed from scratch. In most cases, equationoriented modeling languages (Pantelides, 1988; Barton and Pantelides, 1994) are employed in this case. Efficiency is increased significantly as compared to template based coding of process unit subroutines (Kröner et al., 1990), but there is still lacking support indicated by a number of interviewees.

\subsubsection{Conceptual modeling and model representation}

The development of a conceptual model is a highly creative and often intuitive task. Keeping the purpose of the model in mind, the modeler and the cooperating domain experts scan the process for phenomena that might be of interest in some kind of brainstorming approach. It is important to keep track of all, even the most exotic, ideas for possible later use for model refinement. Experienced modelers will more rapidly focus on the relevant phenomena governing the process behavior. This "filtering" stage is typically supported by literature search and crude calculations.

Along with the identification of important phenomena some model structure is defined. The structuring is either oriented at the signal flow if the target is a block diagram type model (the control engineer's approach), or it is oriented at some physical entities like apparatus, physical phases etc. of the process if the target is some type of compartment model (the chemical engineer's approach). The latter dominates within the group of interviewees. Often, a top-down approach is chosen, where a coarse model structure is gradually refined by decomposition of already introduced parts. However, in all cases bottom-up elements are always mixed in. An important example is the development and analysis of a particle model (a catalyst particle, a polymer solution droplet, etc.) in a disperse process.

The choice of states in every model compartment is important to obtain a good abstraction. The experienced modeler has an eye for choosing the "correct" states. None of the interviewees mentioned explicitly to structure this choice by use of thermodynamic concepts like intensive and extensive variables.

Some people work (at least implicitly) with check lists which include a sequence of frequently asked useful questions to guide the decision on the most important model characteristics. Some kind of standardized model building blocks (see 
(Marquardt, 1996) or (Drengstig et al., 1997) for examples) may support the derivation of a proper process abstraction. However, such a standard is nonexisting in industrial modeling teams. One interviewee stated that it would only be accepted if defined and enforced during the education of the modeler or by some external consultant.

The simpler the better has been stated as a general rule for choosing a conceptual model in many interviews. Typically, the modeler only includes the phenomena (s)he is certain about. However, in case of explicitly designing models for reuse (as it is the case for every model library of a dedicated simulator), there is an incentive to increase generality (and hence complexity) of the model. In these cases, all phenomena anticipated possibly significant in future application not known at present are included and parameterized.

On the basis of these preliminary considerations, a hypothesis comprising structure and relevant phenomena together with the underlying assumptions is formed. Most often, this step is done unsystematically in an implicit rather than an explicit manner. Instead, experienced modelers seem to use some kind of pattern matching approach to directly form this hypothesis. They seem to use a solution to a previous somehow similar problem, though not referenced explicitly, for guidance in the sense of case-based reasoning. This hypothesis can unfortunately be tested only by completing and evaluating the resulting model at a much later stage.

The degree of formalization and documentation of conceptual modeling is very different and depends very much on the personality and background of the modeler. Some emphasize for example the necessity to clearly separate conceptual modeling from model equation development while others unify both phases and directly try to come up with a set of equations.

The former group tends to derive explicit conceptual models using text and informal or semiformal figures before deriving model equations. The representation of the conceptual model is o often close to the presentation used in the target modeling environment. Frequently used graphical representations are some kind of either individually defined 'simulator-relevant P\&ID', which may also be set up in a hierarchical manner to assist model refinement, or the presentation icons used in some favored simulation tool. Bond graphs (Gawthrop and Smith, 1996) are also used in some rare cases.

The latter group does not distinguish between the conceptual modeling and model equation phases. We have seen modelers in favour of both approaches even though the latter constitutes the majority group. To distinguish further, the more experienced a modeler is in some application domain, the less (s)he is explicit in deriving a conceptual model as an intermediate stage towards the model equations. However, members of this group also indicate the risk of sloppiness, if a conceptual model is not derived and documented in a systematic manner.

A couple of the interviewees underline that an explicit conceptual model can be helpful in a dia$\log$ ue with domain experts. This is due to the fact that many domain experts are not used to read and think in partial differential-algebraic equations. Rather, they think in engineering concepts which are typically captured in a graphical and/or phenomema-based description.

It has become quite clear that conceptual modeling and the formulation of model equations is often not separated but highly intertwined, though all the interviewees have distinguished the two tasks to a lesser or higher extent. In simple cases, equation patterns are just copied (or written down without reflection) and the equation terms are specialized. In more difficult and uncommon situations, the equations are rederived from some basic principles. Some modelers write equations on paper before coding them, whereas others directly write equations in the modeling language of an equation-oriented simulator instead. The development of equations is considered error prone by many of the interviewees in all but the frequently occurring standard cases. On the other hand, if a complete conceptual model (including structure, phenomena and state variables) is available, the development of model equations seems to be straightforward.

The favored approach also seems to depend on the modelers' background. For example, control engineers tend to think about simple block diagram models first, or mathematicians typically think in and rely on equations to a much higher extent than chemical engineers who think in engineering concepts rather than equations.

\subsubsection{Implementation and verification}

Implementation and verification is obviously effectively supported if block-oriented simulators with good model libraries are employed.

In case of equation-oriented modeling languages the implementation and debugging (in the sense of syntax checking) is very similar to software development. Most interviewees do not view this as being a major task. Modularization is extensively used to manage complexity. Modelers like to transfer the conceptual model structure to the structure of the implemented model code. They argue that some but not sufficient assistance is 
provided for this transfer by current modeling environments.

Some modelers rearrange the equation set before coding in order to improve robustness and efficiency during the numerical solution. Important issues are proper scaling, elimination of linear equations in the equation set or creating linear equations by introducing auxiliary variables for strongly nonlinear problems, and reformulation of nonlinear terms. In differential-algebraic problems some modelers try to eliminate algebraic equations to the extent possible for robustness reasons. The index of a dynamic model is typically not considered explicitly, because higher index model formulations are usually (implicitly) avoided by an experienced modeler. Further, if an index problem occurs, the simulation system would flag a warning message.

Verification, i.e. a check whether the coded model reflects the intent of the modeler and ultimately the requirements formulated in the functional specification, is seldom performed. Rather, the model is run and the simulation results are checked for plausibility by the modeler her/himself. This situation is viewed as becoming more critical in face of quality control issues.

Debugging aids of current simulators are characterized as useful though not perfect. In particular, the link between numerical problems and physical assumptions coded in the equations is mentioned to be missing. Some modelers increase model complexity during a large number of modeling refinement cycles to effectively support debugging in extreme situations since small incremental changes guarantee transparency and therefore facilitate error identification.

\subsubsection{Initialization}

While for steady-state flowsheeting packages powerful special-purpose initialization procedures are available, the general facilities offered by current process modeling environments (Pantelides, 1988; Kröner et al., 1990; Barton and Pantelides, 1994) provide much less support. Therefore, model initialization has a strong influence on the modeling process.

The most common approach for getting around initialization problems is to apply an evolutionary model development approach by using a simpler model version to initialize a more complex one. As a consequence, many more model versions are created during model development than it would be the case if model initialization was less difficult. Furthermore, this approach results in a high number of iterations between the tasks conceptual modeling, model representation, implementation, and initialization.
In addition to this evolutionary approach tearing and homotopy methods are applied if they are offered by the modeling environment considered. Further methods for getting around initialization problems are much less systematic and highly specific to a special environment.

\subsubsection{Validation}

All interviewees state that validation is extremely important, and, in particular, they emphasize that model validation is a task that grows larger than initially anticipated. Model validation is often impeded for several reasons. First, there is a lack of time (up to 30-50 per cent of the total time spent for the project) and financial resources to collect the data necessary on lab or production scale processes. Moreover, the interpretation of the measurements requires intimate knowledge of the process and the instrumentation. Second, model validity often is implicitly determined by the functional specification of the model-based application. As an example let us consider model predictive control where it is the prediction properties of the model on the optimization horizon that are all important. Specifications of these properties must be inferred from the performance properties on the model predictive control application. No systematic methods seem to be available for this purpose at the moment.

Ideally, a validation procedure should work bottomup. Every basic building block of a simulation model (i.e. phenomenological correlations such as reaction kinetic expressions, the fluid phase of a reactor, or the reactor itself) should be compared with experimental data from carefully designed experiments. Typically, such an approach can only be carried out in the laboratory. There is obviously a significant effort involved, which is often not spent due to time and budget constraints.

Alternatively, the simulation model can be validated on a coarser scale by using the available (and possibly a small number of additional) measurements on the real process. Validation of steady-state process flowsheet models is routinely applied in industrial practice. Often dedicated validation tools are employed instead of general purpose modeling and simulation systems. At least coarse activity models or check lists seem to be available for this purpose in the various companies.

In contrast, there seems to be no systematic validation procedure for rigorous nonstandard steadystate unit models or nonlinear dynamic models in place at the moment. Unsurprisingly, there is also a lack of flexibility and functionality in current tools to perform validation of very detailed steady-state or first principles based dy- 
namic models in an efficient way. Hence, rigorous validation of such models is rarely undertaken at the moment. Typically, a number of steadystate operating points or a single time series (i.e. a step response) near a single operating point is compared with the model. Some parameters are adjusted (in most cases manually) to make the model match the experimental data.

If no experimental data are available (for example during a process design project) the simulation results may be at least audited by an expert team. The team members check results with their expectation based on their process knowledge and previous experience. No systematic procedure of experimenting with the model in order to guarantee the inspection of all important modes seems to be available for this purpose. If a steady-state simulation is available, the dynamic simulator's predictions are compared to those of the steadystate simulator in steady-state.

\subsubsection{Documentation and reuse}

Documentation is considered important since reuse of the same or a similar model is occurring more often than expected. This means that earlier developed models, either inhouse or from other available sources, often form a basis for new models. Despite its importance, many of the interviewees claim that they don't find sufficient time for proper documentation due to the fact that there are always more good projects than human resources. Further, they state, documentation is less fun than problem solving, and management does not sufficiently honour proper documentation in practice.

There seems to be a difference in people's attitude towards documentation or even between different companies' strategy. In some cases there is virtually no documentation provided at the end of a project, whereas in other cases a significant amount of time is used for documentation. The latter is especially true if documentation is formalized as a part of the company's or division's quality assurance procedures.

Documentation of the final model would definitely help, but is not sufficient. What is really needed is the sequence of important model versions developed during one project together with their assessment, as well as a documentation of the critical choices made during the model development phase, in particular the assumptions on which the model is based. If not explicitly stated, these assumptions and choices may not be obvious to a person who wants to utilize the model in future modeling projects. Hence, the explicit documentation of assumptions and choices is a critical success factor to enable efficient reuse of existing models.

From the interviews, we found that one or several of the following three types of documentation are produced in current practice: (i) inline in model code, (ii) documentation for end user or client, and (iii) documentation on model development and application including rationale and unsuccessful trials. As to (iii) some state that this is documented in a comprehensive manner while others state that it is difficult to update information on model development because of its unstructured and iterative nature. Documentation often deteriorates over time because of a lack of updating.

It is interesting to note, that some of the interviewees started to build up their personal model library. However, all of them gave up since the lack of time and the missing tool support prevented the build-up and maintenance of a well-documented personal library of reasonable coverage. Rather, parts of existing models of her/his own are reused in later projects. These models are almost never exchanged between members of some modeling team in an operating company.

\subsection{Life-cycle perspectives}

In practice, it is very difficult to maintain models over the life cycle of a plant. On the one hand this is due to the documentation problem as described above. On the other hand, rapidly advancing simulation technology renders the model code written in the representation format of some modeling environment almost useless over time. In order to prevent this problem, the knowledge associated with the model must be captured, rather than the mere code in some language. Ideally, the documentation should completely define all the activities and the rationale which lead to the model employed. In this case, the model can be reengineered easily at a later time.

Models are only maintained if considered economically important. Maintenance of on-line applications is done by dedicated personnel who are extremely familiar with the particular plant. Maintenance of plant models on a larger scale seems to be infeasible at the moment since the plant personnel are not sufficiently trained to maintain models. Some companies have started to build technology groups which among other things maintain the models of the plant. In contrast to operating companies, vendor and engineering companies have a more elaborate and clearly defined strategy on model reuse. They tend to build a model library aiming at a significant reduction of modeling in later projects by configuring and parameterizing submodels. This observation is further supported by the fact that these companies 
may develop relatively general submodels. As an example a dynamic model of an oil/gas separator may be used for both two and three-phase separation, and for different geometric structures.

Because of the lacking documentation and the heterogeneous modeling platforms almost no reuse of models happens over the process life cycle at the moment. In many cases a model is simply rewritten instead of reengineering an existing model from a previous life cycle phase.

Some interviewees claim that modeling should start much earlier in the process life cycle. It would be appropriate to use modeling techniques already during chemical research or during benchscale process development at the latest. All the information gathered should be collected and stored in a structured manner in some model base spanning the whole life cycle from chemical research until process operation. There is, however, a lack of optimism on achieving this. The reasons are twofold: first, current technology does not offer adequate support to accomplish this economically and second, there are no serious management incentives yet pushing the life-cycle perspective on a company wide and division encompassing way.

To focus on process design the use of dynamic simulators is not considered to be viable at present due to two reasons: (i) The configuration of a dynamic model and simulation scenarios is very time-consuming compared to steady-state simulators. Hence, in face of the continuously decreasing duration of the design phase, model development efficiency needs to be improved significantly. (ii) Steady-state simulators are considered robust and relatively easy to use by a broad group of process engineers because of easily accessible processoriented manuals. Dynamic simulators are not yet accepted by many process engineers due to a lack of reliability, robustness and user-friendliness.

\subsection{General aspects of industrial process modeling}

All interviewees agree that to a high percentage standard steady-state simulations are carried out. But the use of nonstandard detailed steady-state and, in particular, dynamic models is steadily increasing. Some interviewees link this increase of nonstandard models to company strategy in which the use of models, stated more or less explicitly, is seen as an important means for improved operations.

The operating companies perform model development both inhouse and in cooperation with external companies. Confidentiality and the fact that model development is seen as a means for process knowledge improvement limits the use of external resources in some cases. However, most of the interviewees in the operating companies predicted an increasing demand for external modeling services in the future. A view promoted by vendors is that these companies play an instrumental role in spreading the use of dynamic models in particular for improving process operations and for training applications.

Several of the interviewees state that model development cost is a major hurdle for an increase in the use of models. This is also true, to a lesser extent however, for modeling software cost. Further, they emphasize the importance of having a diversified set of model types as a means to reduce cost. In particular, it has often been mentioned that simple block diagrams like largely empirical models do very well in many (in particular control related) applications. One interviewee states, for example, that empirical models can be developed at about $\frac{1}{10}$ of the development cost for mechanistic models. It is, however, difficult to assess the overall benefit of an empirical model because much less new process knowledge is developed compared to first principles based modeling. Typically, such a model cannot be used as a basis for later applications.

The cost of model development hinders the spreading of model-based process engineering techniques. This is particularly true for small scale processes since the reduction of production cost is relatively small compared to large scale processes. One of the interviewees mentioned a figure of $90 \%$ of total cost spent for model development in a plant-wide optimization project. He also explains that an on-line model application often cannot be economically justified for medium capacity (and turnover) chemical processes in contrast to large capacity petrochemical and refinery processes.

At the moment, one modeler is typically working on his own on a certain modeling problem. However, he works on various projects. The number of projects being active at the same point in time may reach the order of 15 . Some interviewees expect process modeling to evolve more and more towards a team activity due to the increasing size of the projects and due to tighter time constraints. This development towards a multi-project team based approach to the development of process models and model-based applications is expected to significantly influence the modeling process as a whole.

Still, the modeling experts of the research centres face significant difficulties in communicating with the plant personnel or other domain experts since there is a continuing large gap in education in the area of modeling and model-based applications between the two groups. Time consuming discussions and public relations campaigns illustrating successful applications are required to explain and 
promote the technology to potential clients. Any means to make the model and the results of model application more transparent to plant operating personnel will help. More severe, it must be conjectured that significant potential for process improvement is not taken advantage of because of the lacking feeling for anticipating potential benefit for model-based solutions. This problem can only be solved in the longer run by extending and improving education in modeling and simulation as well as model-based applications.

\subsection{Wish list}

At the end of each interview the interviewees were invited to suggest improvements on todays modeling practice and tools. The need for a variety of improvements has been emphasized by almost all the interviewees. Most of their suggestions are categorized in Table 3.

\section{DISCUSSION}

\subsection{The modeling process revisited}

The objective of the empirical investigation has been to improve our understanding of the modeling process, at least on the coarse level of granularity as reflected by the initial task structure presented in Section 2.3. Further, we were interested in identifying at least to some extent a network of subtasks for key modeling tasks such as the development of the conceptual model or the set of model equations. We are going to discuss to what extent this expectation has been met.

The existence of the initially defined tasks has been confirmed during the interviews. However, the modeling process is by no means linear. As expected a priori, it is characterized by extensive iterations. Moreover, the modeler does not necessarily jump between neighboring tasks (in the sense of the sequence defined in Section 2.3). Further, there was no clear pattern of iterations in the interviews indicating the diversity of different modelers' work process. Hence, we are not able to deduce a prescriptive modeling process on the level of granularity introduced in Section 2.3 which would be acceptable by all the interviewees. Nevertheless, there are some interesting findings.

There is a very close link between some tasks. The groups (i) problem understanding, problem statement including functional specification, initial data collection and tool selection, (ii) conceptual modeling and model representation (by means of equations for example), (iii) implementation and verification, (iv) initialization and debugging, and (v) validation have been identified. The
Table 3. Suggestions for improving current modeling technology.

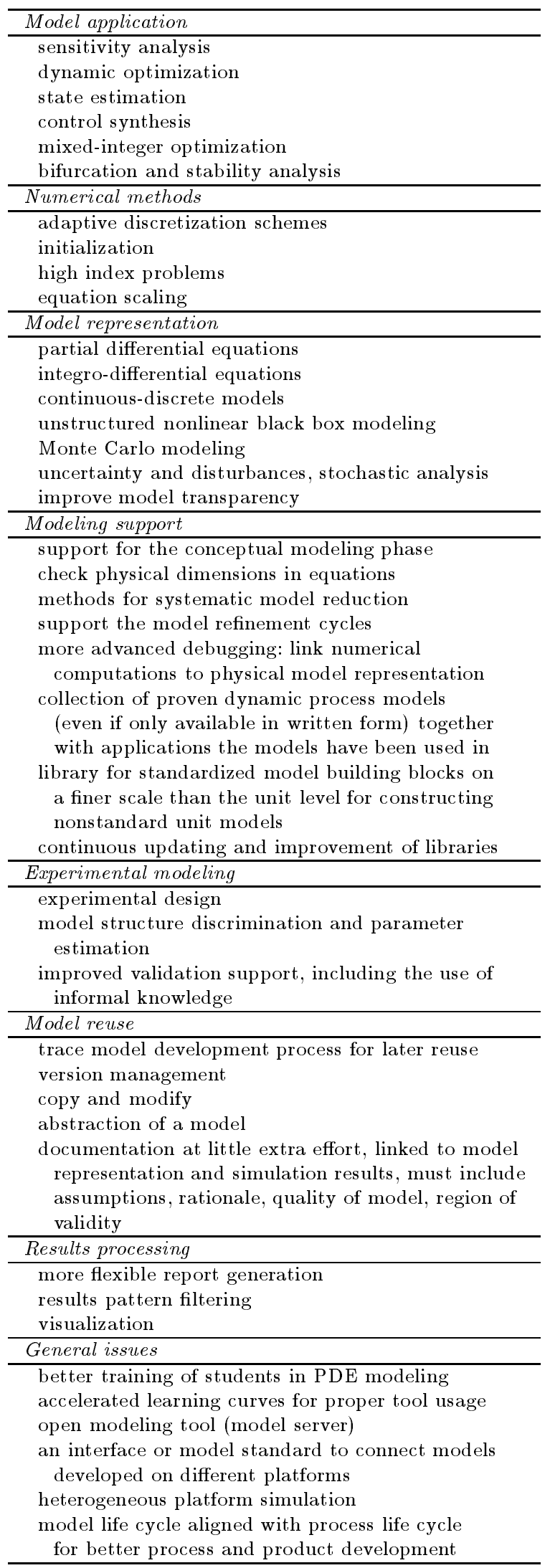

documentation task (vi) is essentially linked to all of the other task groups with different emphasis. The degree of distinction between the tasks within 
such a group heavily depends on the modeler's preferences.

An experienced modeler moves a lot between the tasks (and within the task on the subtask level). He does not rely on a simple sequence with a small number of well-defined recycles such as a less experienced modeler might do. However, the experienced modeler, on a whole, moves along the task groups (i), (ii), (iii), (iv), and (v) sequentially in the sense of a moving window capturing more than one task group at a time. As the modeling process goes by, the degree of back-steppings to the early task groups diminishes in favour of the forward steppings to the later task groups. Nevertheless, there exist numerous iterations between the task groups rendering a highly interwined and complex modeling process. In principle, there is a link between all the tasks on the granularity level suggested in Section 2.3 resulting in a fully connected network.

A transparent modeling process can therefore not be formulated on this granularity level from the interview information. In contrast to earlier publications (Marquardt, 1996; Denn, 1985), we conjecture in summary, that this is impossible in principle. The coarse tasks of Section 2.3 correspond more or less to unstructured containers of subtasks of a finer granularity and contain even not well-understood subtasks.

The understanding of the modeling process must therefore target at a finer level of granularity. However, it is very difficult to identify the fine granular modeling steps by our case study approach chosen. This is due to the fact that real expert modelers (as selected for our interviews) do not necessarily have to think about their mode of operation during model development. They just do it according to an implicit and hidden work process (like everybody drives a car without reflection). Some of the interviewees, however, indicated that they have thought about some parts of the modeling process in detail for one reason or another. Extensive discussions on conceptual modeling strategies, on bottom-up vs. top-down, on pattern matching approaches and the like have convincingly shown that there is a chance to identify modeling steps of fine granularity if an appropriate methodology is employed.

The modeling process is implicit and highly iterative and, hence, somewhat unstructured. Further, increased experience tends to promote this unstructuredness. From an objective standpoint it can be debated whether this unstructuredness really improves the efficiency of the modeling process. It might well be the case that an emphasis on structure will actually promote model development efficiency among experienced modelers.

\subsection{The fundamental research issue}

These findings lead us to the fundamental key research topic of identifying a model of the modeling process ${ }^{1}$. This ambitious long-term research goal is motivated by the following hypothesis: A significant improvement in any design activity characterized by a large degree of creativity can only be achieved if the design process in itself is sufficiently understood and formalized in the sense of some model. Hence, it is not sufficient to use the result of the activity (the mathematical process model). Since the selected modeling tool heavily influences the modeling process, as identified from the interviews, it seems to be of great importance.

The required identification of a model of the modeling process is comparable to the identification of a mathematical model even though the model formats will be significantly different. As in mathematical modeling, we may distinguish two complementary approaches - a data driven and a first principles driven approach. The data driven approach relies on detailed empirical studies of real modeling processes in an industrial environment. The information gathered that way cannot be interpreted (as also learned in our study) if no initially postulated (partial) model of the modeling process based on preliminary insight is employed. The empirical data is analyzed to identify patterns which support or falsify the initial model. From a practical point of view, the identification of the modeling process must be aided by computerbased modeling support tools which offer some functionality to record all the actions of an expert modeler during a real project. Further, these tools have to provide functionality for analyzing such a trace (namely an instance of the modeling process) based on the a priori model of the modeling process. This interpretation step leads to an a posteriori refinement of the model and forms the basis for an extension of the modeling tool for the next iteration cycle. That way, the degree of generality and coverage of the model of the modeling process will gradually evolve.

In order to start with this procedure, it is suggested to apply some ideas on the fundamental characteristics of design processes as developed in software engineering (Pohl, 1996) and summarized as follows. An instance of a modeling process or of any other design process (Fig. 1), as actually employed in a certain modeling project, comprises a complex sequence of consecutive elementary or aggregated modeling steps (Jarke and Marquardt, 1996; Lohmann and Marquardt, 1996; Marquardt, 1996). Every modeling step is closely

\footnotetext{
1 Note that the modeling process is part of an even more complex design process which aims at some model-based application such as the design and implementation of an on-line optimization system.
} 


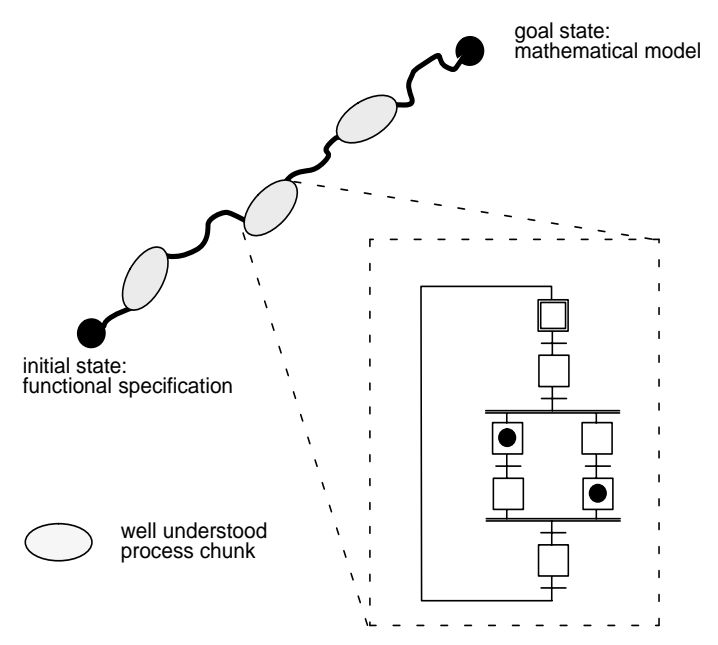

Fig. 1. Well understood chunks of the modeling process.

linked to its product (the mathematical model) and to the decisions taken by the members of the development team. Process steps, products, and decisions must be structured to handle complexity by means of some generic concepts and represented by some formalism amenable to computerbased reasoning. Some of the modeling steps, socalled process chunks are well understood. Examples of process chunks are the derivation of balance equations from a certain set of specifications or a degrees of freedom analysis. These process chunks can be carried out automatically or by intervention of an expert modeler if certain conditions are fulfilled. Such a situated action (Jarke and Marquardt, 1996) is typically preceded by a poorly understood sequence of creative modeling steps which are carried out according to the individual preference of an expert modeler in an unforeseeable manner.

Obviously, the sketched empirical and constructive approach can only be viewed as a long term (and probably visionary) research goal. Short to mid term milestones need to be defined and accomplished towards this goal not only for increasing the success probability but also for improving current modeling practice immediately. Concrete research questions and advanced modeling tools requirements are summarized in the following section.

\subsection{Research agenda}

Advanced modeling tools should provide some simple means of supporting the modeling process in the sense of the last section. An initial and exploratory attempt is currently made along these lines by the authors (Jarke and Marquardt, 1996; Dömges et al., 1996; Bogusch et al., 1996). As a general guideline for tool development, no strict control flow can be imposed on the modeler. The tool must offer the flexibility to the modeler to focus on whatever issue he is interested in at a certain time. The modeler must have access to the functionality (s)he needs at a given step. The information entered into the system during a particular modeling step must be propagated in the system to be used in other modeling steps at a later time regardless the sequence these modeling steps are carried out. As an example, information from a conceptual modeling tool must be transferred to the documentation tool and vice versa.

Any well-understood part of the modeling process should be identified as a process chunk. These chunks must be precisely defined, formalized and finally implemented in the modeling tool. The availability of these chunks to the user will improve efficiency and hopefully quality of the modeling process.

Subsequently, a number of key requirement for advanced modeling tools are stated. Most of them are originating from the interviews, but their concrete definition has also been influenced by the authors' views on the subject. The list concentrates on issues directly related to the modeling process. Hence, issues related to the use of models in an application, e.g. the derivation of a state estimator, an optimization algorithm, etc. is not included.

(1) Storing and retrieving initial data and functional specification. All the data collected and the decisions taken during the formulation of the functional specification should stored to be retrievable at later stages of the modeling process. We envision hypertext like systems where different kinds of documents, graphs, experimental data etc. can be stored in a network of labeled linked information nodes (Pohl, 1996; Westerberg, 1996), and an gIBIS-like decision editor to capture the rationale of functional specifications (Jarke and Marquardt, 1996; King and BañaresAlcántara, 1996).

(2) Model base for structured model representation. Model building blocks of various kind and granularity for structure and physicochemical phenomena modeling should be provided in a library (Marquardt, 1991; Stephanopoulos et al., 1990). Governing equations with underlying assumptions and typical use cases (parameter values, examples, etc.) should be included. Support for continuous updating and improvement of the modeling concepts library would be appreciated. Case-based generalization should be envisioned in the long run to partially automate the extension of the library. 
(3) Modeling on the knowledge level. With increasing emphasis of the development of nonstandard unit models, better support for conceptual modeling and equation development is required. Besides the model base discussed above suitable user interfaces including selective browsers should be provided to effectively search in and navigate through the library.

(4) Support of the model refinement cycle. Supporting the modeling process just for one particular application is considered a reasonable starting point for covering the whole modeling process during the plant life cycle. This would also cover the refinement and extensions typically made during initialization of a complex model.

(5) Integration of data driven and first principles based modeling. There is an incentive to integrate mechanistic and empirical modeling (Johansen and Foss, 1997) since this enables the modeler to use process knowledge and informative process data efficiently. There is very little support for adapting such models in todays tools. Methods and tools for data handling, time series analysis, data filtering, parameter estimation and model structure discrimination as well as experimental design are required.

(6) Validation. Model validation is closely related to empirical, identification based modeling techniques. Methodologies for validation of complex nonlinear (dynamic) models need to be developed and appropriate tool functionality must be provided. It should be possible to include informal knowledge provided by plant operators.

(7) Support for documentation. The additional effort for producing the documentation must be kept at a minimum. In particular, mechanisms for documenting the modeling rationale, i.e. the assumptions and critical choices incorporated in a model, the different model versions developed during a project as well as the model quality and the model validity should be provided. This information is vital for efficiently supporting model reuse.

(8) Model interpretation. A family of related models is needed during different life cycle phases. Hence, a model server (Marquardt, 1991; Pantelides and Britt, 1994) comprising some symbolic model representation deduced from the model base mentioned in item (2) should be employed to provide suitable views on a symbolic multi-faceted model (the steady-state or the dynamic version, a causeand-effect graph, the symbolic Jacobian of the right hand sides etc.) to some clients which do the simulation or any other type of analysis in an open environment. This way, several applications could be linked to the same process model.

(9) Model integration. There is an incentive for integrating models. They could be of the same formalism but developed by means of different tools. Further, they could be even of different formalisms such as black box linear models, neural net models and first principles based models. Typically, models of different formalisms stem from different modeling environments. Integration could be on the model representation level by means of product data modeling techniques (McKay et al., 1996) or open model representation languages (Elmqvist et al., 1997), or on the procedural level where complete simulators are integrated and coordinated during runtime in the sense of heterogeneous platform simulation (Brüll et al., 1997). Component ware (Adler, 1995) could be a useful technology for systems implementation.

(10) Collaborative model development. Since there seems to be a distinct trend towards larger modeling projects, team work methodologies (Spurr et al., 1994) should be adopted in the modeling process and supported by future tools.

\section{CONCLUSIONS}

The industrial field study, which is a very untypical research approach in chemical engineering, but has been used in other areas like software engineering, revealed some interesting information on the industrial modeling process. It is not possible to define a transparent model of the modeling process on the coarse level of granularity defined before conducting the interviews. Instead those chunks of the modeling process that are well understood have to be identified and described by an appropriate model of the modeling process. These process chunks should describe work processes like model initialization which increase the productivity of industrial modelers. Advanced modeling tools are required providing means for supporting the modeling process and allowing the adaption of the modeling process to the user's needs.

\section{Acknowledgements}

We appreciate the cooperation of our industrial colleagues. Without their dedication and their open mind this study would not have been possible. In particular we thank R. Bachmann, T. Christensen, E. Dikow, E. Gran, R. Guth, H. Hartmann, M. Hillestad, U. Kuhn, A. Mjaavatten, U. Nieken, R. Perne, S. Scholl, K. Strand, S. Sælid, S.Wasbø, P.Wynn for the interviews. 


\section{REFERENCES}

Adler, R.M. (1995). Emerging standards for component software. IEEE Computer 28, 68-77.

Aris, R. (1991). Manners makyth modellers. Trans. IChemE 69, 165-174.

Barton, P.I. and C.C. Pantelides (1994). Modeling of combined discrete/continuous processes. AICHE J. 40, 966-979.

Bogusch, R., B. Lohmann and W. Marquardt (1996). Computer-aided process modeling with MODKIT. In: Proc. Chemputers Europe III. McGraw Hill. Frankfurt.

Brüll, L., F. Hubbuch and S. Quest (1997). Process simulation on heterogeneous networks. Computers chem. Engng. Submitted for publication.

Carroll, J.M. (1995). Scenario-Based Design. Wiley. New York.

Curtis, B., H. Krasner and N. Iscoe (1988). A field study of the software design process for large systems. Communications of the $A C M$ 31, 1268-1287.

Denn, M.M. (1985). Process Modeling. Longman. New York.

Dömges, R., K. Pohl, M. Jarke, B. Lohmann and W. Marquardt (1996). PRO-ART/CE An environment for managing the evolution of chemical process simulation models. In: Proc. 10th European Simulation Multiconference (A. Javor, A. Lehmann and I. Molnar, Eds.). pp. 1012-1017. Society for Computer Simulation International. Budapest.

Drengstig, T., S. Wasbø and B.A. Foss (1997). A formal graphical based process modeling methodology. In: Proc. PSE/Escape 7. Trondheim, Norway. Accepted for publication.

Elmqvist, H., A. Jeandel, S.E. Mattson, P. Sahlim and M. Otter (1997). Modelica - language design for multi-formalism modeling. ESPRIT Project Simulation in Europe Basic Research Working Group. http://www.Dynasim.se/Modelica/.

Finkelstein, A., J. Kramer and B. Nuseibeh (1994). Software Process Modeling and Technology. Wiley. New York.

Gawthrop, P. and L. Smith (1996). Metamodelling: Bond Graphs and Dynamic Systems. Prentice Hall. London.

Hammer, M. and J. Champy (1995). Business Reengineering. Campus. Frankfurt.

Jarke, M. and W. Marquardt (1996). Design and evaluation of computer-aided process modeling. In: Proc. 1st Int. Conf. on Intelligent Systems in Process Engng ISPE'95. AICHE Symposium Series No. 312 (J.F. Davis, G. Stephanopoulos and V. Venkatasubramaniam, Eds.). Vol. 92. pp. 97-109. CACHE Publications. Danvers, MA.

Johansen, T. A. and B. A. Foss (1997). Operating regime based process modelling and identifi- cation. Computers and Chemical Engineering 21, 159-176.

King, J.M.P. and R. Bañares-Alcántara (1996). The extension and evolution of the process design representation. Computers chem. Engng 20, S171-S176.

Kröner, A., P. Holl, W. Marquardt and E.D. Gilles (1990). DIVA - An open architecture for dynamic simulation. Computers chem. Engng 14, 1289-1295.

Ljung, L. (1987). System Identification: Theory for the User. Prentice-Hall. Englewood Cliffs, NJ.

Lohmann, B. and W. Marquardt (1996). On the systematization of the process of model development. Computers chem. Engng 20, S213S218.

Marquardt, W. (1991). Dynamic process simulation - recent trends and future challanges. In: Chemical Process Control CPC-IV (Y. Arkun and W.H. Ray, Eds.). pp. 131-180. CACHE Publications. New York.

Marquardt, W. (1996). Trends in computer-aided modeling. Computers chem. Engng 20, 591609.

McKay, A., M.S. Bloor and A. de Pennington (1996). A framework for product data. IEEE Trans. on Knowledge and Data Engng 8, 825838.

Oakland, J.S. (1989). Total quality management. In: Proc. 2nd Int. Conf. on Total Quality Management. pp. 3-17. Cotswold Press.

Pantelides, C.C. (1988). Speedup - recent advances in process simulation. Computers chem. Engng 12, 745-755.

Pantelides, C.C. and H.I. Britt (1994). Multipurpose process modeling environments. In: Foundations Computer-Aided Design Conf. FOCAPD'94. pp. 128-141. CACHE Publications. Snowmass, CO.

Pohl, K. (1996). Process Centered Requirements Engineering. Wiley. New York.

Spurr, K., P. Layzell, L. Jennison and N. Richards (1994). Computer Supported Cooperative Work. Wiley. New York.

Stephanopoulos, G., G. Henning and H. Leone (1990). Model.la. A language for process engineering. Part I and II. Computers chem. Engng 14, 813-869.

Westerberg, A. (1996). Distributed and collaborative computer-aided environments in process engineering design. In: Proc. 1st Int. Conf. on Intelligent Systems in Process Engng ISPE'95. AICHE Symposium Series No. 312 (J.F. Davis, G. Stephanopoulos and V. Venkatasubramaniam, Eds.). Vol. 92. pp. 184-194. CACHE Publications. Danvers, MA.

Yin, R.K. (1984). Case Study Research - Design and Methods. Sage Publications. London. 
